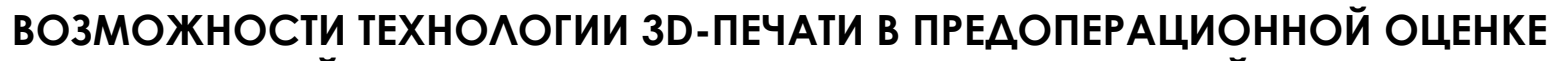 ОСОБЕННОСТЕЙ АНАТОМИИ АЕФЕКТА МЕЖЖЕАУАОЧКОВОЙ ПЕРЕГОРОАКИ И АНОМААИИ РАЗВИТИЯ ПРАВОГО ЖЕАУАОЧКА}

\author{
Завадовский К.В.' ', Кривощеков Е.В.2, Бразовский К.С. ${ }^{3}$, лежнев А.А. ${ }^{2}$, \\ Мочула А.В. 1, Шмакова Н.А. 2, Скодренко И.Н.4, Василькова К.Н.5, МиШкина А.И. 1 , \\ Саушкин В.В.1 , Янулевич О.С. 2 , Фелоров В.В.6
}

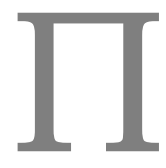

риведено клиническое наблюдение ребенка 11-ти месяцев, у которого врожденный дефект межжелудочковой перегородки сочетался с наличием аномальных мышечных тяжей в полости первого желудочка, что приводицо к формированию "двухкамерного" правого желудочка и создавало трудности с определением правильной тактики хирургического мечения.

В представценном наблюдении приводятся данные МСКТ сердца с контрастированием, на основании которых была сформирована физическая трехмерная модель сердца, позволившая кардиохирургам выбрать оптимальный доступ и вид коррекции порока. В обсуждении представлены исторические и современные аспекты применения технологии 3D в медицине.

Ключевые слова: МСКТ сердца, дефект межжелудочковой перегородки, дополнительная полость сердца, 3D-печать.

Контактный автор: Завадовский К.В., e-mail: konstzav@gmail.com

Для иитирования: Завадовский К.В., Кривощеков Е.В., Бразовский К.С., Аежнев А.А., Мочула А.В., Шмакова Н.А., Скофенко И.Н., Василькова К.Н., Мишкина А.И., Са ушкин В.В., Янулевич О.С., Федоров В.В. Возможности технологии 3d-печати в предопераиионной оиенке особенностей анатомии дефекта межжелудочковой перегородки и аномалии развития правого желудочка. REJR 2018; 8(1):194-201. DOI:10.21569/22227415-2018-8-1-194-201.
Статья получена:
29.01.2018
Статья принята: 28.02.2018

1- НИИ кардиомогии, Томский национальный исследовательский медицинский центр РАН Маборатория радионуклидньх методов исследования. г. Томск, Россия. 2 - НИИ кардиологии, Томский национальньй исследовательский медицинский центр РАН. Отделение сердечнососудистой хирургии. г. Томск, Россия. 3 - ФГБОУ ВО «Сибирский государственный медицинский университет" Минздрава РФ. Кафедра медицинской и биологической кибернетики с курсом медицинской информатики. г. Томск, Россия. 4 - ГБУЗ "Детская городская клиническая больница №13 им. Н.Ф. Фиматова" ДЗ.

г. Москва, Россия.

5 - ГБОУ Аицей №1535. г. Москва, Россия. 6 - Национальньй исследовательский Томский политехнический университет. Научнопроизводственная маборатория "Современные производственные технологии" Инженерной школы новьх производственных технологий. г. Томск, Россия. 


\section{D PRINTING IN PREOPERATIVE ASSESSMENT OF CARDIAC ANATOMY IN PATIENT WITH CONGENITAL VENTRICULAR SEPTAL DEFECT AND DOUBLE-CHAMBERED RIGHT VENTRICLE}

\author{
Zavadovsky K.W. 1, Krivoshekov E.V. 2, Brasovsky K.S. 3, Lezhnev A.A. 2, Mochula A.V. 1, \\ Shmakova N.A. 2, Skofenko I.N. 4, Vasilkova K.N. ${ }^{5}$, Mishkina A.I ., Saushkin V.V. 1, \\ Yanulevich O.S. 2, Fedorov V.V.6
}

\section{I}

$\mathrm{n}$ this report, the case of 11 months old child is presented. This patient suffered from congenital ventricular septal defect. Moreover, he had double-chambered right ventricle. It determines difficulties in surgical treatment strategy. We presented cardiac MSCT data, which allow creating 3D model of the heart. It allowed choosing optimal surgical treatment tactic. In the discussion, the historical and modern data about abilities of 3D printing in medicine is presented.

Keywords: cardiac MDCT, ventricular septal defect, double-chambered right ventricle, three-dimensional printing

Corresponding author: Zavadovsky K.W., e-mail: konstzav@gmail.com

For citation: Zavadousky K.W., Krivoshekov E.V., Brasousky K.S., Lezhnev A.A., Mochula A.V., Shma-kova N.A., Skofenko I.N., Vasilkova K.N., Mishkina A.I1., Saushkin V.V., Yanulevich O.S., Fedorov V.V. 3D printing in preoperative assessment of cardiac anatomy in patient with congenital ventricular septal defect and double-chambered right ventricle. REJR 2018; 8(1):194-201. DOI:10.21569/2222-7415-2018-8-1-194-201.

Received: 29.01.2018 Accepted: 29.02.2018
1 - Nuclear department, Cardiology Research Institute, Tomsk National Research Medical Centre, Russian Academy of Sciences. Tomsk, Russia. 2 - Department of Cardiovascular Surgery, Tomsk National

Research Medical Centre, Russian Academy of

Sciences. Tomsk, Russia. 3 - Department of Medical and Biological Cybernetics with a Course in Medical Informatics, Siberian State Medical University. Tomsk, Russia.

4 - N.F. Filatov Children's City Clinical Hospital № 13. Moscow, Russia. 5 - Lyceum №1535. Moscow, Russia. 6 - National research Tomsk Polytechnic University.

Tomsk, Russia.
A

номальные мышечные тяжи камер сердца, приводящие к формированию дополнительных полостей правого жемудочка, - достаточно редкая врожденная аномамия [1]. Данная патология встречается как изолированно, так и в сочетании с размичными врожденными пороками сердца, чаще всего с дефектами межжелудочковой перегородки разцичной мокализации [2]. Дия выбора последующей тактики хирургического мечения сопутствующих врожденных дефектов важно понимание топографии внутрисердечных структур в условиях аномальной геометрии помости правого желудочка. Важную роль в этом играют современные методы неинвазивной диагностики, которые позволяют получить двух- и трёхмерные модеми сердца [3]. В то же время, даже качественные трехмерные модели основных структур сердца не всегда позволяют кардиохирургам определиться с тактикой операции. В этих ситуациях прибегают к созданию физических трёхмерных моделей.

\section{Кинническое набиюдение.}

Пациент К., 11 месяцев, в декабре 2016 года в плановом порядке поступиц в НИИ кардиологии Томского НИМЦ с диагнозом врожденный порок сердца: дефект межжелудочковой перегородки (ДМЖП).

При поступлении жалобы на потмивость, снижение аппетита, плохую прибавку в весе. Общее состояние ребенка удовлетворительное. При аускультации мегких дыхание пуэримьное, проводится по всем отделам, хрипов нет. ЧДД в покое 36 в минуту. Тоны громкие, ритмичные, систолический шум над всей поверхностью сердца. ЧСС 128 в минуту. АД на руке 100/86 мм рт. ст., SpO2 на руках - 82-89\%. Пульс на мучевых и бедренных артериях симметричный, удовлетворительного наполнения. Рост 81 см. Вес $9 \mathrm{kr}$.

Из анамнеза известно, что врожденный порок сердца (ДМЖП) бым диагностирован внутриутробно. В периоде новорожденности ребенок перенес острую цитомегаловирусную инфекцию (получал мечение до 2,5 месяцев). В кардиохирургическом отдемении по месту житемьства 16.03.2016 года ребенку была выполнена операция суживания мегочной артерии.

По данным эхокардиографии выявлена диматация всех камер сердца, кроме мевого предсердия. Крупный мышечный среднеапикальный ДМЖП. Надклапанное сужение мегочной артерии. Сужение между трабекулярной и отточной частями правого желудочка с формированием дополнительной полости.

Дия уточнения анатомии правого желудочка, оценки взаимоотношений между допо нительным мышечным гребнем правого желу- 
дочка (ПЖ), который формировал добавочную полость, и выводным трактом ПЖ, а также дия оценки топографии и взаимоотношения с указанными структурами дефекта межжекудочковой перегородки, пациенту была выполнена MCKT сердца с контрастированием.

Параметры сканирования: ретроспективная ЭКГ-синхронизация; напряжение на трубке $80 \mathrm{kB}$, сима тока 200-250 мА (с ЭКГ модумяцией), скорость вращения трубки 0,38 с, питч: 0,20:1. Для контрастирования коронарных артерий использовали внутривенную инфузию 13 м $\Lambda$ Йопамидома (370 мг йода/м^) (Йопамиро, Bracco, Италия) со скоростью 2 м $/$ с.

MCKT сердца: сердце увеличено в размерах за счет правых отделов. Отмечается гипертрофия задней и боковой стенок правого желудочка с наличием гипертрофированного мышечного гребня, идущего от заднебоковой стенки правого желудочка к межжелудочковой перегородке. Толщина мышечного гребня составяяет 6 мм. Краниально данный мышечный гребень участвует в формировании задней стенки выводного тракта правого желудочка. Кроме того, имеется второй мышечный гребень, располагающийся справа от трикуспидального клапана, толщина его составляет 8 мм. Каудально данный мышечный гребень соединяется со стенкой правого желудочка. Данные мышечные гребни формируют "дополнительную мышечную полость", имеющую сообщение с правым желудочком и выраженное трабекулярное строение. В апикальном отделе межжелудочковой перегородки определяется дефект, размерами $11 \times 8,8$ мм. Правое предсердие деформировано, увеличено в размерах, компримировано описанным выше мышечным гребнем, трикуспидальный клапан расположен типично. Аевое предсердие компримировано правым предсердием и смещено вцево.

Иегочной ствоц отходит типично, имеет место надклапанное сужение (состояние после суживания мегочной артерии; 5,4×5,1 мм), раз-

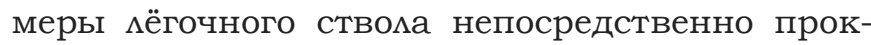
симахьнее бифуркации - 12,5х11,7 мм. Разме-

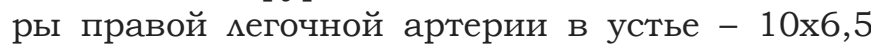
мм, мевой мегочной артерии - 13х11,6 мм.

Коронарные артерии отходят типично. Правая коронарная артерия "зажата" между стенкой правого предсердия и дополнительной камерой правого желудочка.

После проведения МСКТ сердца с контрастированием у кардиохирургов остались вопросы относительно анатомии расположения дефекта межжелудочковой перегородки и достижимости через конвенциональные хирургические доступы в межжелудочковой перегородке.

В связи с этим было принято решение о создании физической трехмерной модели сердца данного ребенка.
ДАя построения трехмерной модеми было использовано свободно распространяемое программное обеспечение Slicer 3D версии 4.6 с дополнительными модуяями собственной разработки для сегментации исходного изображения [4].

Предварительная обработка данных MCKT сердца заключалась в выделении области интереса и выравнивании гистограммы по яркости в пределах выдеменного участка. После допо нительной ручной коррекции контраста и яркости для получения наилучшего раздемения фона и изображения анатомических структур, значения яркости вокселей изображения были преобразованы в формат с двойной точностью (тип double) для дальнейших вычислений.

На первом этапе построения модеми была осуществлена сегментация изображения с разделением на отдемьные ткани и определение границ. Быц применен комбинированный алгоритм сегментации по пороговым значениям $о$ гарифма яркости вокселей, совмещенный с методом деформируемых поверхностей дия одновременного определения границ с использованием оператора Sobel. Дия повышения пространственного разрешения в методе деформируемых поверхностей использована технология субвоксельной интерполяции, при этом интерполированное пространственное разрешение составицо 0,25 мм по всем координатам.

Основной проблемой при реконструкции трехмерных моделей на основе МСKTизображений является сложная топология внутренней поверхности сердца, что приводит к появлению дефектов поверхности (незамкнутость, появление несуществующих отверстий, слишком тонких стенок и других) и погрешности геометрических размеров. При объемной визуализации это не имеет большого значения, однако для виртуального планирования хирургического вмешательства и трехмерной печати необходимо обеспечить выполнение формальных требований к геометрии внутренних и наружных поверхностей.

Дия решения указанной проблемы были применены модули собственной разработки для реализации комбинированного метода сегментации с одновременным выделением границ объектов и субвоксельной интерполяцией обнаруженных границ. В автоматическом режиме было правильно распознано около 95\% всей обмасти интереса. Слабо контрастированные обмасти изображения внутри желудочков и вблизи межжелудочковой перегородки были сегментированы вручную.

На основе сегментированного изображения была построена трехмерная модемь в виде триангулированной поверхности наружной поверхности и внутренних полостей сердца (рис. 1). Полученная модемь была экспортирована в 
формате STL для дальнейшей предпечатной подготовки в среде MeshMixer компании Autodesk [5]. Подготовка заключалась в анализе поверхности, заполнении дефектов и последующего снижения размерности (децимации). На этом этапе также проводился анализ модеми на предмет ее пригодности к печати расплавленным пластиком (fused deposition modelling, FDM). Важнейшим требованием к предпечатной подготовке явцяется сохранение максимально точной формы поверхностей и формирование зон поддержки участков, не опирающихся на предыдущие слои. Дия доступа к внутреннему объему модемь была раздемена на три части (рис. 2).

Экспериментальным путем были подобраны оптимальные параметры для трехмерной печати: толщина слоя не более 0,1 мм, скорость перемещения экструдера при печати не более 20 мм/с, обязательно охлаждение. Время печати модели составило 18 часов.

На полученной модеми удалось детально рассмотреть анатомические особенности инфундибулярного отдела правого желудочка, стала понятна мокализация дефекта межжелудочковой перегородки относительно фиброзного кольца трикуспидального клапана и невозможность выполнения пластики ДМЖП доступом через правое предсердие (рис. 3, 4). Стало очевидным, что для выполнения пластики ДМЖП необходимо проведение вентрикулотомии в строго определенном месте.

Такой вид диагностики позволим на дооперационном этапе опредемиться с хирургической стратегией, тщательно отработать последовательность действий при оперативном вмешательстве и избежать возможных осложнений.

Пациенту выполнена операция: пластика дефекта межжелудочковой перегородки дакроновой заплатой, ушивание дефекта межпредсердной перегородки, миотомия выходного отдела правого желудочка, пластика выходного отдела правого желудочка заплатой Gore-Tex, удаление суживающей тесьмы с мегочной артерии в условиях ИК и КХКП. Имплантация противоспаечной заплаты PRECLUDE.

\section{Обсуждение.}

История 3D-печати началась в 1983 г., когда Чарльз Хам разработал и запатентовал технологию "стереолитографии" (SLA) дмя печати 3D-объектов по данным цифровых модемей из фотополимеризующихся композитных материамов. В 1987 году компанией 3D Systems Corp бым создан первый стереолитографический аппарат дмя послойного создания (выращивания) физического объекта по цифровой 3D-модели с помощью особого устройства - 3D-принтера [4]. Впрочем, сам термин "3D-печать" появицся только в 1995 году в Массачусетском технологическом институте. Несмотря на большую за- интересованность научного мира столь ценным изобретением, дальнейшее развитие технологии несколько застопорилось - 3D-принтерам не хватало мощности, они демали всё медменно и неточно, после печати изделия приходимось дорабатывать напильником в прямом смысле этого слова [5].

Широкое распространение технология 3Dпечати получима с 2010 года, благодаря значительному снижению цены на 3D-принтеры изза высокой конкуренции на рынке. С этого времени наблюдается неукцонный рост с тенденцией в сторону снижения цен на принтеры и расходные материалы примерно в 10 раз каждые 2 года. В 2012 году рынок 3Dпринтеров составим 2,2 млрд. \$, что на 29\% выше 2011 года. K 2016 году рост рынка достиг до 6 блн. \$ (из которых $11 \%$ - это медицинское использование) и, по прогнозам агентства Bloomberg, в 2022 году превысит 26 блн. \$ [6, 7].

За последние годы интерес к 3D-печати в медицине увеличивается в геометрической прогрессии. Это заметно и по числу, и по качеству докладов на крупнейших мировых радиологических конгрессах. В докладе руководителя маборатории 3D-печати Питтсбургского университета д-ра Аниш Годадра, представценном на конгрессе RSNA в 2016 г., сформулированы основные условия получения качественных биомедицинских издемий с использованием техномогии 3D-печати. В первую очередь, это получение данных компьютерной томографии высокого качества. При создании 3D-модеми важна каждая деталь, поэтому рекомендовано высокое пространственное разрешение детектора и проведение КТ-исследования с толщиной среза менее 1.25 мм. Обработку полученных данных домжен проводить опытный врач мучевой диагностики, имеющий гмубокое понимание анатомии в данной области. Точная сегментация объекта имеет ключевое значение [8].

Особое место эксперты в области 3Dпечати уделяют самообразованию рентгенологов. Специализированные курсы проводятся ежегодно в рамках конгресса RSNA, на базе всемирно известных кАиники Мейо и Массачусетского Технологического Университета [9].

Третий этап - создание стереолитографического (STL) файла. Дия него необходимо $\Lambda и-$ цензированное медицинское программное обеспечение. Потери анатомических сегментов (что возможно в неспециализированных дия медицины программах) не допускаются [10, 11]. Необходимо отметить, что с 2016 года рабочая станция Volume Share 7 позволяет пользоватемям конвертировать данные DICOM в стереолитографический фай (формат .stl) двумя кАиками. Конвертация выполняется с помощью специализированного медицинского програм- 


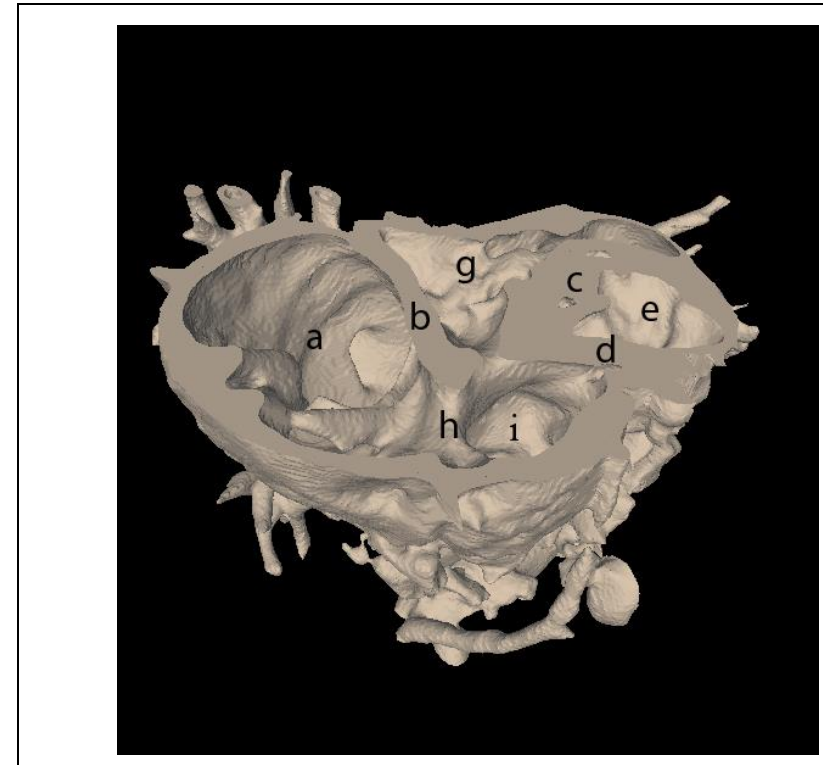

Рис. 1 (Fig. 1)

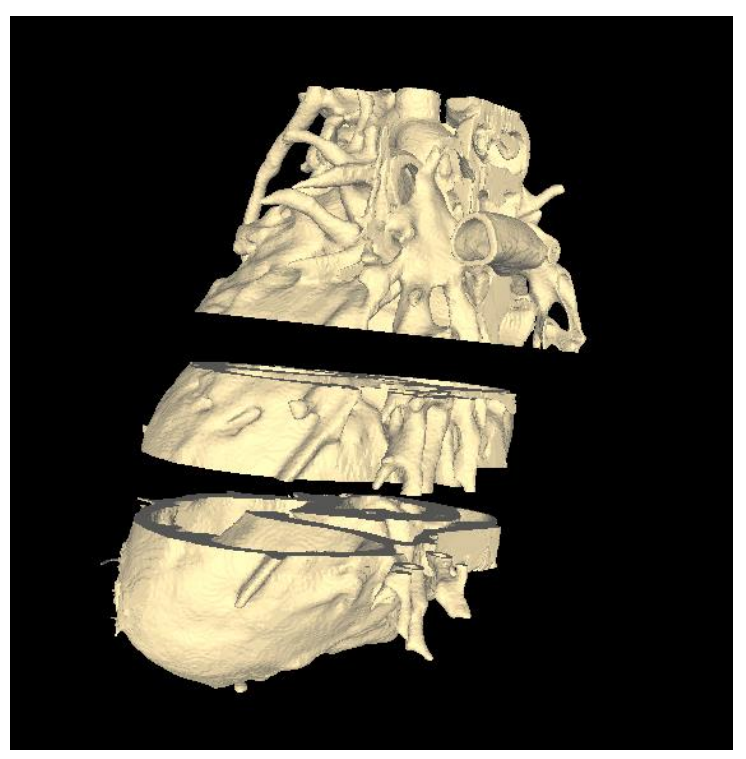

Pис. 2 (Fig. 2)
Рис. 1. 3D-реконструкция серАца.

Триангулированные поверхности после реконструкции.

Fig. 1. 3D heart reconstruction.

Triangulated surfaces after reconstruction.

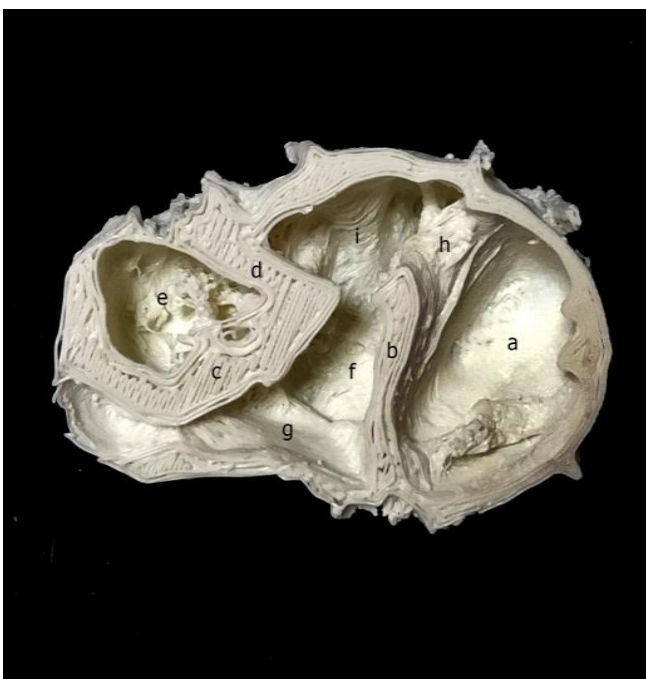

Рис. 3 a (Fig. 3 a)
Рис. 2. 3D-реконструкция.

Модель сердца посме предпечатной подготовки.

Fig. 2. 3D heart reconstruction.

Heart model after prepress preparation.

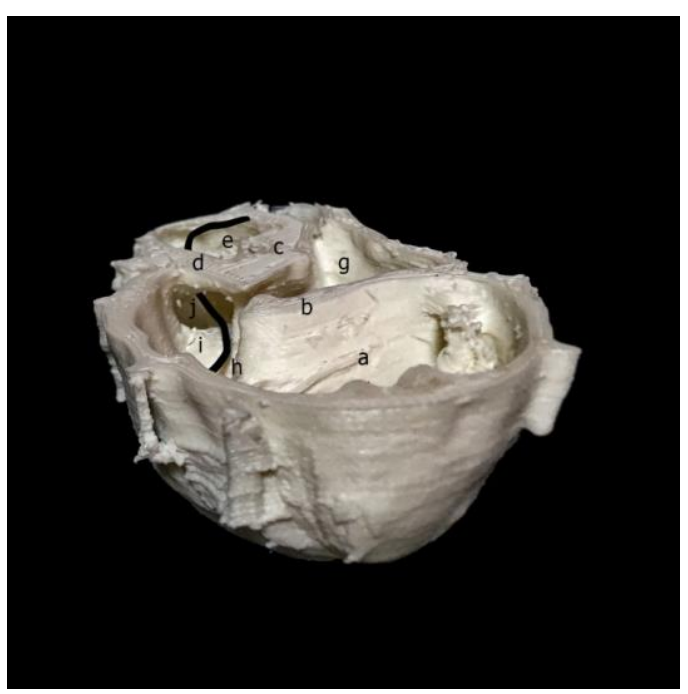

Pис. 36 (Fig. 3 в)

Рис. 3. 3D-реконструкция серАца.

$\mathrm{a}-$ Поперечный срез 3D-модели сердца на уровне дефекта межжелудочковой перегородки. Визуализируется дополнительная мышечная полость (е) и дефект межжелудочковой перегородки (h).

б - Проекция с наилучшей визуализацией коммуникации (j) между аномальной дополнительной полостью (е) и полостью ПЖ (i). ДАя наглядности из дополнительной полости через коммуникацию в полость ПЖ введен черный проводник.

\section{Fig. 3. 3D-reconstruction of the heart.}

a - Transverse slice of 3D model of the Heart (at the level of ventricular septum). Additional muscular chamber (e) and ventricular septal defect $(\mathrm{h})$ are nicely shown.

$\mathrm{b}$ - The projection of 3D heart model with the best visualization of the communication (j) between the anomalous chamber (e) and the right ventricular cavity (i). The black wire is introduced through communication into the right ventricular chamber. 
ного обеспечения GE Healthcare и поставцяется в составе базовой конфигурации рабочей станции AW.

И, конечно, сама 3D-печать. Правильный выбор 3D-принтера и материала для печати, в зависимости от планируемой области испоцьзования [12].

Использование технологии 3D-печати в медицине стало развиваться всего несколько мет назад. Однако технология настолько быстро вошла в ежедневную практику врачей, что в 2017 г. американское управцение по санитарному надзору за качеством пищевых продуктов и медикаментов (FDA) опубликовало официальные рекомендации по производству издемий медицинского назначения с использованием 3D-принтеров, включающие детализированные требования к техническим характеристикам устройств для 3D-печати, процессу их тестирования и требованиям к качеству [13].

Основными направлениями явцяются стоматология, ортопедия и травматология, а также кардио- и нейрохирургия $[14,15]$.

3D-модели помогают докторам при планировании хода операции и в оптимизации хи- рургического процесса, дают возможность в сложных ситуациях избежать возможных осложнений, незаменимы при обучении момодых специалистов [16 - 18].

3D-модеми сердца, напечатанные в реальных размерах, служат тренажерами, на которых кардиохирурги отрабатывают этапы операции в каждом сложном и нетипичном случае, и даже могут помочь в подборе донорского сердца в зависимости от веса пациента (что особенно важно в детской кардиохирургии) [19 $-21]$.

Значительно проще и нагляднее становится общение с пациентом или родителями (в детской хирургии), обсуждение анатомических особенностей врожденного порока сердца (ВПС), мибо другой патоцогии, а также хода операции. Такой подход явцяется шагом к персонифицированному мечению каждого пациента [22].

Технологии 3D-печати активно развиваются в России. Новый проект по 3D-печати чемовеческого сердца стартовал на базе научнообразовательного центра "Современные производственные технологии" Национального иссле-

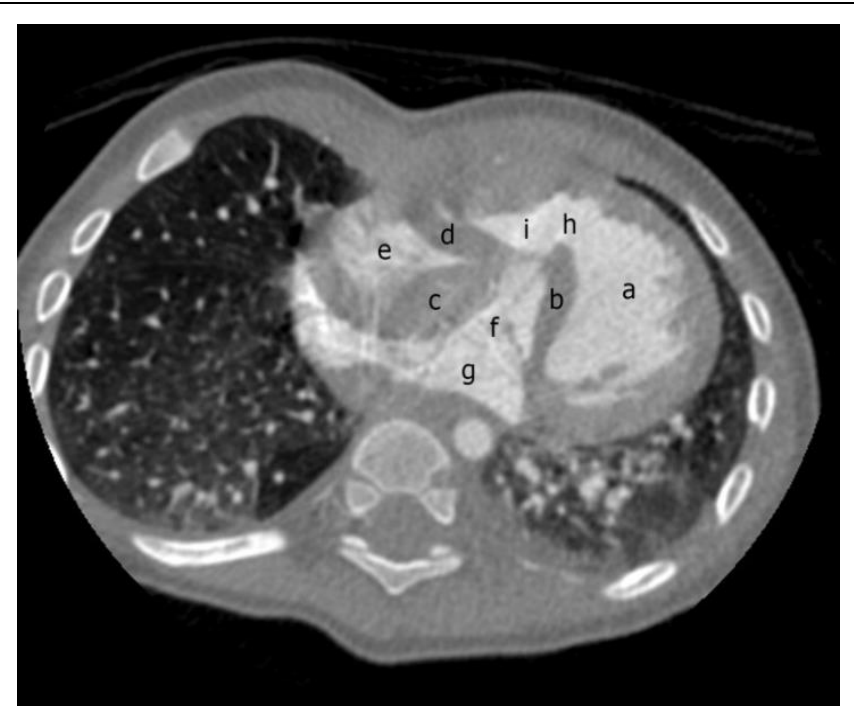

Puc. 4 a (Fig. 4 a)

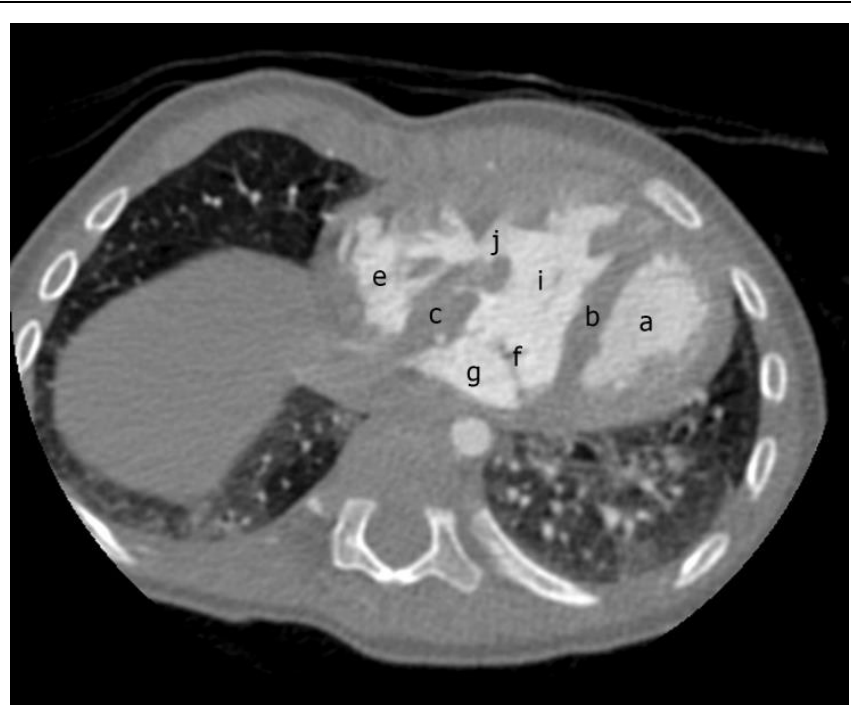

Рис. 4 б (Fig. 4 в)

\section{Рис. 4. МСКТ органов груАной полости. Аксиальная плоскость.}

а - поперечный срез на уровне дефекта межжелудочковой перегородки. Визуализируется дополнительная мышечная полость (е) и дефект межжелудочковой перегородки (h).

б - поперечный МСКТ срез на уровне коммуникации между аномальной дополнительной полостью и полостью ПЖ. Визуализируется сообщение (j) между дополнительной мышечной камерой (е) сердца с полостью правого жекудочка (i).

\section{Fig. 4. MSCT, heart. Axial slice.}

a - transverse cardiac MSCT slice at the level of ventricular septum. Additional muscular chamber (e) and ventricular septal defect (h) are nicely shown.

b - transverse cardiac MSCT slice at the level of the communication between the anomalous chamber and the right ventricular cavity. The communication (j) between the additional right ventricular chamber (e) and the right ventricular cavity (i) is nicely shown. 
довательского Томского политехнического университета. Политехники выполняют его дия Томского НИИ кардиологии. Сотрудники центра печатают пластиковые копии настоящих сердец на основе данных компьютерной томографии. Имея в руках осязаемую модемь, кардиохирурги смогут подробнее изучать дефекты органа и выбирать подходящие способы оперативного мечения. Первые 3D-модели взрослого человеческого сердца были напечатаны учеными ТПУ в конце 2016 года [23].

\section{Закиючение.}

В данном клиническом наблюдении представлено редкое наблюдение пациента с дефектом межжелудочковой перегороди в сочетании с аномальной дополнительной полостью правого желудочка. Показано, что своевременное вы-

\section{Cписок митературы:}

1. Animasahun B.A., Ekure E.N., Njokanma O.F. Doublechambered right ventricle: an uncommon congenital heart disease. Case report and literature review. Cardiovascular Journal of Africa. 2011; 22:274-277.

2. Kharwar R.B., Dwivedi S.K., Sharma A. Double-Chambered Right Ventricle with Ventricular Septal Defect and Subaortic Membrane - Three-Dimensional Echocardiographic Evaluation. Echocardiography. 2016; 33:323-327.

3. Завадовский К. В., Панькова А. Н. Сиинтиграфическая оиенка дисфункиии правого желудочка сердиа у паииентов с тромбоэмболией легочной артерии. Медииинская визуализаиия. 2009; 3:24-30.

4. Fedorov A., Beichel R., Kalpathy-Cramer J., Finet J., FillionRobin J-C., Pujol S., et al. 3D Slicer as an Image Computing Platform for the Quantitative Imaging Network. Magnetic Resonance Imaging. 2012; 30 (9): 1323-1341.

5. Meshmixer is state-of-the-art software for working with triangle meshes (2018). Available at: https://www.meshmixer.com/ (accessed 29 January 2018).

6. Изобретатель технологии 3D-печати Чарльз Халл номиниован на премию European Inventor Award: [Электронный ресурс] // 3D-принтеры сегодня, 2013-2016. URL: http://3dtoday.ru/industry/izobretatel-tekhnologii-3d-pechaticharlz-khall-nominirovan-na-premiyu-european-inventoraward.html (Дата обращения: 29.01.2018).

7. От пиииы до автомобиля: 8 ошеломительных примеров того, что можно напечатать на 3D-принтере: [ЭлектронHый pecypc] Magnis.news, 2009-2018. URL: http://www.magnis.news/rus/news/8933/ (Дата обpaщeния: 29.01.2018).

8. Wohlers 2017 Report on 3D Printing Industry Points to Softened Growth (2017). Available at: http://www.rapidreadytech.com/2017/04/wohlers-2017-

report-on-3d-printing-industry-points-to-softened-growth/ (accessed 29 January 2018).

9. GE Keeps Its Checkbook Handy After All-in Bet on 3-D Printing (2017). Available at: https://www.bloomberg.com/news/articles/2017-04-20/gekeeps-its-checkbook-handy-after-all-in-bet-on-3-d-printing (accessed 29 January 2018). полнение пациенту МСКТ сердца с дальнейшим созданием физической трехмерной модеми позволило выбрать наиболее правильную хирургическую тактику для коррекции порока. Более широкое использование технологий 3D-печати в сфере кардиологии и сердечно-сосудистой хирургии будет способствовать мучшей подготовке кадров, мучшему взаимопониманию между врачами и пациентами, а также позволит оптимизировать мечебную тактику. интересов.

Источник финансирования и конфмикт

Авторы данной статьи подтвердили отсутствие финансовой поддержки исследования и конфцикта интересов, о которых необходимо сообщить.

10. Tips for implementing $3 D$ printing at your hospital (2015). Available

http:/ / www.auntminnie.com/ index.aspx?sec=sup\&sub=adv\&p $a g=$ dis $\&$ ItemID $=112380$ (accessed 29 January 2018).

11. How radiologists can optimize their role in $3 D$ printing (2017).

http://www.auntminnie.com/index. aspx?sec $=\sup \& s u b=a d v \& p$ ag $=$ dis\&ItemID $=118441$ (accessed 29 January 2018).

12. The power and promise of $3 D$ printing: Part 1 (2016). http:/ / www.auntminnie.com/index. aspx?sec $=\sup \& s u b=a d v \& p$ ag $=$ dis\&ItemID=113863 (accessed 29 January 2018).

13. The power and promise of $3 D$ printing: Part 2 (2016). http://www.auntminnie.com/index. aspx?sec=sup\&sub=adv\&p ag $=$ dis\&ItemID=113881 (accessed 29 January 2018).

14. 4 steps to high-quality $3 D$ printing for radiology (2016). http://www.auntminnie.com/index. aspx?sec=sup\&sub=adv\&p $a g=$ dis\&ItemID $=116130$ (accessed 29 January 2018).

15. FDA releases guidance on $3 D$ printing of medical products (2017).

http:/ / www.auntminnie.com/index. aspx?sec=sup\&sub=adv\&p ag=dis\&itemid=119319 (accessed 29 January 2018).

16. 3D-printed model helps prepare for stroke clot removal (2017).

http://www.auntminnie.com/index. aspx?sec $=\sup \& s u b=a d v \& p$ $a g=$ dis\&ItemID $=118424$ (accessed 29 January 2018).

17. 15. Решетов И.В., Святославов Д.С., Кудрин К.Г., Дуб. В.А., П.А. Медведев. Технологическое обеспечение аддитивных технологий для реконструкиии лииевого скелета. REJR 2017; 7 (4): 140-153. DOI:10.21569/2222-7415-2017-7-4-140153.

18. 3D printing shortens hip surgery times, lowers costs (2017). http:/ / www.auntminnie.com/index. aspx?sec $=\sup \& s u b=a d v \& p$ ag $=$ dis $\& I t e m I D=118004$ (accessed 29 January 2018).

19. The power and promise of $3 D$ printing: Part 1 (2016). http://www.auntminnie.com/index. aspx?sec=sup\&sub=adv\&p $a g=$ dis\&ItemID=113863 (accessed 29 January 2018).

20. Researchers launch library of $3 D$ heart models (2016). http://www.auntminnie. com/index. aspx?sec $=\sup \& s u b=a d v \& p$ $a g=$ dis $\&$ ItemID $=103158$ (accessed 29 January 2018).

21. 3D models put pediatric hearts in surgeons' hands (2015). 


\section{RUSSIAN ELECTRONIC JOURNAL OF RADIOLOGY}

http://www.auntminnie.com/index.aspx?sec $=s u p \& s u b=m r i \& p$ $a g=$ dis \&ItemID $=111955$ (accessed 29 January 2018).

22. Funding, expertise give Jump an edge on $3 D$ hearts (2016). http://www.auntminnie.com/index. aspx?sec $=s u p \& s u b=a d v \& p$ $a g=$ dis\&ItemID $=115267$ (accessed 29 January 2018).

23. 3D images aid selection of heart transplant donors (2015). http://www.auntminnie.com/index.aspx?sec $=s u p \& s u b=a d v \& p$ ag $=$ dis\&ItemID $=112641$ (accessed 29 January 2018).

24.Anwar S, Singh GK, Varughese J, Nguyen H, Billadello JJ, Sheybani EF, et al. $3 D$ Printing in Complex Congenital Heart

\section{References:}

1. Animasahun B.A., Ekure E.N., Njokanma O.F. Doublechambered right ventricle: an uncommon congenital heart disease. Case report and literature review. Cardiovascular Journal of Africa. 2011; 22:274-277.

2. Kharwar R.B., Dwivedi S.K., Sharma A. Double-Chambered Right Ventricle with Ventricular Septal Defect and Subaortic Membrane- Three-Dimensional Echocardiographic Evaluation. Echocardiography. 2016; 33:323-327.

3. Pankova A.N., Zavadousky K.V. Estimation of Dysfunction of the Hearts's Right Ventricle at Patients with Pulmonary Embolizim by Scintigraphy. Medicinskaya vizualizaciay. 2009; 3:2430 (in Russian).

4. Fedorov A., Beichel R., Kalpathy-Cramer J., Finet J., FillionRobin J-C., Pujol S., et al. 3D Slicer as an Image Computing Platform for the Quantitative Imaging Network. Magnetic Resonance Imaging. 2012; 30 (9): 1323-1341.

5. Meshmixer is state-of-the-art software for working with triangle meshes (2018). Available at: https://www.meshmixer.com/ (accessed 29 January 2018).

6. Inventor of $3 D$ printing technology Charles Hull is nominated for the European Inventor Award (2014). Available at: http://3dtoday.ru/industry/izobretatel-tekhnologii-3d-pechaticharlz-khall-nominirovan-na-premiyu-european-inventoraward.html (accessed 29 January 2018) (in Russian).

7. From pizza to car: 8 stunning examples of what you can print on a 3D printer (2016). Available at: http://www.magnis.news/rus/news/8933/ (accessed 29 January 2018) (in Russian).

8. Wohlers 2017 Report on 3D Printing Industry Points to Softened Growth (2017). Available at: http://www.rapidreadytech.com/2017/04/wohlers-2017-

report-on-3d-printing-industry-points-to-softened-growth/ (accessed 29 January 2018).

9. GE Keeps Its Checkbook Handy After All-in Bet on 3-D Printing (2017). Available at: https://www.bloomberg.com/news/articles/2017-04-20/ge-

keeps-its-checkbook-handy-after-all-in-bet-on-3-d-printing (accessed 29 January 2018).

10. Tips for implementing $3 D$ printing at your hospital (2015). Available

http://www.auntminnie.com/index.aspx?sec $=s u p \& s u b=a d v \& p$ $a g=$ dis\&ItemID $=112380$ (accessed 29 January 2018).

11. How radiologists can optimize their role in $3 D$ printing (2017).

http://www.auntminnie.com/index.aspx?sec $=s u p \& s u b=a d v \& p$ $a g=$ dis\&ItemID=118441 (accessed 29 January 2018).

12.The power and promise of $3 D$ printing: Part 1 (2016). http://www.auntminnie.com/index.aspx?sec $=s u p \& s u b=a d v \& p$
Disease: Across a Spectrum of Age, Pathology, and Imaging Techniques. JACC Cardiovascular Imaging. 2017; 10(8):953956. doi: 10.1016/j.jcmg.2016.03.013.

25. Томские ученые печатают 3D-модели детских сердеи для кардиохирургии: [Электронный ресурс] // 3Dpulse.ru, 2014-2018.

URL:

http://www.3dpulse.ru/news/meditsina/tomskie-uchenyepechatayut-3d-modeli-detskih-serdets-dlya-kardiohirurgii/

(Дата

обрашения:

29.01.2018)

$a g=$ dis\&ItemID $=113863$ (accessed 29 January 2018).

13. The power and promise of 3D printing: Part 2 (2016). http://www.auntminnie.com/index. aspx?sec $=s u p \& s u b=a d v \& p$ $a g=$ dis \&ItemID $=113881$ (accessed 29 January 2018).

14. 4 steps to high-quality $3 D$ printing for radiology (2016). http:/ / www.auntminnie.com/index.aspx?sec $=s u p \& s u b=a d v \& p$ $a g=$ dis\&ItemID $=116130$ (accessed 29 January 2018).

15. FDA releases guidance on $3 D$ printing of medical products (2017).

http://www.auntminnie.com/index.aspx?sec=sup\&sub=adv\&p ag=dis\&itemid=119319 (accessed 29 January 2018).

16. 3D-printed model helps prepare for stroke clot removal (2017).

http:/ / www.auntminnie.com/index. aspx?sec=sup\&sub=adv\&p $a g=$ dis\&ItemID $=118424$ (accessed 29 January 2018).

17. Reshetov I.V., Svyatoslavov D.S., Kudrin K.G., Dub V.A., Medvedev P.A. Technological supply of additive technologies for face skeleton reconstruction. REJR 2017.7(4):140-153. DOI:10.21569/2222-7415-2017-7-4-140-153 (In Russian).

18. 3D printing shortens hip surgery times, lowers costs (2017). http://www.auntminnie.com/index. aspx?sec=sup\&sub=adv\&p ag $=$ dis\&ItemID $=118004$ (accessed 29 January 2018).

19. The power and promise of 3D printing: Part 1 (2016). http:/ / www.auntminnie.com/ index. aspx?sec $=\sup \& s u b=a d v \& p$ ag $=$ dis $\& I t e m I D=113863$ (accessed 29 January 2018).

20. Researchers launch library of $3 D$ heart models (2016). http://www.auntminnie.com/index. aspx?sec $=\sup \& s u b=a d v \& p$ ag $=$ dis $\&$ ItemID $=103158$ (accessed 29 January 2018).

21. 3D models put pediatric hearts in surgeons' hands (2015). http://www.auntminnie.com/index. aspx?sec=sup\&sub=mri\&p $a g=$ dis\&ItemID $=111955$ (accessed 29 January 2018).

22. Funding, expertise give Jump an edge on $3 D$ hearts (2016). http://www.auntminnie.com/index. aspx?sec=sup\&sub=adv\&p $a g=$ dis\&ItemID $=115267$ (accessed 29 January 2018).

23. 3D images aid selection of heart transplant donors (2015). http://www.auntminnie.com/index. aspx?sec $=$ sup\&sub=adv\&p $a g=$ dis\&ItemID $=112641$ (accessed 29 January 2018).

24. Anwar S, Singh GK, Varughese J, Nguyen H, Billadello JJ, Sheybani EF, et al. 3D Printing in Complex Congenital Heart Disease: Across a Spectrum of Age, Pathology, and Imaging Techniques. JACC Cardiovascular Imaging. 2017; 10 (8): 953956. doi: 10.1016/j.jcmg.2016.03.013.

25. Tomsk scientists print $3 D$ models of children's hearts for cardiac surgery

http://www.3dpulse.ru/news/meditsina/tomskie-uchenyepechatayut-3d-modeli-detskih-serdets-dlya-kardiohirurgii/ (accessed 29 January 2018). 